\title{
Equilibrium distributions of discrete phase type
}

\author{
Xueyuan $\mathrm{Wu} *$ \\ Centre for Actuarial Studies, Department of Economics \\ The University of Melbourne, VIC 3010, Australia
}

\begin{abstract}
It is well known that the family of discrete phase-type distributions is closed under convolutions, mixtures, and the unary 'geometric mixture' operation. In this paper, we show that the equilibrium distributions of this family are again of phase-type. A simple result is obtained for the limiting equilibrium distributions, that is, the equilibrium distributions of any discrete phase-type distribution with an irreducible representation always converge to a geometric distribution. Numerical examples are provided in three different cases. At the end of the paper, a more general type of equilibrium distribution is considered to which a discount factor is introduced.
\end{abstract}

Keywords: Discrete phase-type distribution; Equilibrium distribution; High-order equilibrium distribution; Limiting equilibrium distribution

*Dr Xueyuan Wu: Email: xueyuanw@unimelb.edu.au; Tel.: 61-3-83447939; Fax: 61-383446899 


\section{Introduction}

Phase-type distributions form one of the most general classes of distributions permitting a Markovian interpretation. Formalised introductions for the discrete phase-type distributions date back to mid 1970's, see Neuts [15]. However, most focus has been given to the continuous phase-type distributions. Detailed discussions of continuous phase-type distributions can be found in Neuts [16] and Latouche and Ramaswami [9]. Brief overviews of either discrete or continuous phase-type distributions and their properties can also be found in Asmussen [1], Bobbio et al. [3], Drekic et al. [4] and the references therein.

Discrete phase-type (DPH) distributions constitute a class of distributions on non-negative integer set which seem to strike a balance between generality and tractability. They have rational generating functions and include special cases, geometric, negative binomial, as well as linear combinations (including mixture) and convolutions of these distributions. A DPH distribution inherits the special structure from the Markov property of the underlying discrete-time Markov chain. Moreover, the class of DPH distributions is one of the classes which are dense in the class of all discrete distributions on $\mathbb{N}=\{0,1,2, \ldots\}$ so that any distribution may be approximated arbitrarily closely by a suitable DPH distribution.

It is well known that phase-type distributions are closed under convolutions, mixtures, and the unary 'geometric mixture' operation. Maier and O'Cinneide [13] showed that the continuous class is the smallest family of distributions that is closed under these operations. An analogous result holds for the discrete class. And very recently, Bladt and Nielsen [2] studied moment distributions of continuous phase-type and matrix-exponential distributions and proved that they remain within their respective classes. Motivated by this interesting result and also recent increasing attention given to the equilibrium distributions in the literature, the equilibrium distributions of the DPH family are considered here, aiming to prove the property of closure in this case. Latest references for the equilibrium distributions include Fagiuoli and Pellerey [5, 6], Nanda et al. [14], Hesselager et al.[8], Lin and Willmot $[11,12], \mathrm{Li}[10]$ and the references therein.

We know that a DPH random variable $N$ is the time to absorption in a terminating discrete-time Markov chain $\left\{J_{n}\right\}_{n=0}^{\infty}$ with $m+1$ states, one of which is absorbing, namely 0 . The state space of $\left\{J_{n}\right\}_{n=0}^{\infty}$ is $\{1,2,3, \ldots, m\} \cup\{0\}=E \cup$ $\{0\}$ and the initial distribution is $\left(\alpha_{1}, \alpha_{2}, \ldots, \alpha_{m}, \alpha_{0}\right)$. The transition probability matrix of $\left\{J_{n}\right\}_{n=0}^{\infty}$ is

$$
\left[\begin{array}{cc}
\mathbf{T} & \overrightarrow{\mathbf{t}} \\
\overrightarrow{\mathbf{0}} & 1
\end{array}\right]
$$


where $\overrightarrow{\mathbf{0}}=(0,0, \ldots, 0)_{1 \times m}$.

Let $p(n), n \in \mathbb{N}$, denote the probability function (p.f.) of $N$, then it is called a DPH distribution with representation $(\overrightarrow{\boldsymbol{\alpha}}, \mathbf{T})$, where $\overrightarrow{\boldsymbol{\alpha}}=\left(\alpha_{1}, \ldots, \alpha_{m}\right)$, and $\sum_{i=1}^{m} \alpha_{i}=1-\alpha_{0} \leq 1$. Throughout this paper, $\mathbb{N}=\{0,1,2, \ldots\}$ and $\mathbb{N}^{+}=$ $\{1,2, \ldots\}$. Then we have

$$
p(n)=\overrightarrow{\boldsymbol{\alpha}} \mathbf{T}^{n-1} \overrightarrow{\mathbf{t}}^{\top}, \quad n \in \mathbb{N}^{+},
$$

with $p(0)=\alpha_{0}$. In the above definition, $\mathbf{T}=\left(t_{i, j}\right)_{m \times m}$ is a substochastic matrix and $\overrightarrow{\mathbf{t}}^{\top}=\left(t_{1,0}, t_{2,0}, \ldots, t_{m, 0}\right)^{\top}=(\mathbf{I}-\mathbf{T}) \overrightarrow{\mathbf{1}}^{\top}$, where $\mathbf{I}$ is the $m \times m$ identity matrix and $\overrightarrow{\mathbf{1}}=(1,1, \ldots, 1)_{1 \times m}$. Within this paper, a substochastic matrix means a matrix of that the sum of elements in each row is less than or equal to 1 and there is at least one strictly less case. The distribution function of $N$ is $P(n)=$ $1-\bar{P}(n)=1-\overrightarrow{\boldsymbol{\alpha}} \mathbf{T}^{n} \overrightarrow{\mathbf{1}}^{\top}$ and its probability generating function (p.g.f.) is $\hat{p}(z):=$ $\sum_{n=0}^{\infty} z^{n} p(n)=\alpha_{0}+z \overrightarrow{\boldsymbol{\alpha}}(\mathbf{I}-z \mathbf{T})^{-1} \overrightarrow{\mathbf{t}}^{\top},|z|<1$. Moreover, the $l$-th factorial moment of $N$ has the expression

$$
\mu^{(l)}:=E\left[N^{(l)}\right]=l ! \overrightarrow{\boldsymbol{\alpha}}(\mathbf{I}-\mathbf{T})^{-l} \mathbf{T}^{l-1} \overrightarrow{\mathbf{1}}^{\top}, \quad l \in \mathbb{N}^{+},
$$

where $n^{(l)}=n(n-1)(n-2) \ldots(n-l+1)$ is the $l$-th factorial power of $n$ and $n^{(0)}=1$. Let $\mu=\mu^{(1)}$ denote the mean of $N$.

The rest of the paper is structured as follows. Section 2 introduces the high order equilibrium distributions and shows the property of closure of the equilibrium distributions for the DPH family. Section 3 discusses the limiting equilibrium distribution and shows that the limiting equilibrium distribution of any DPH distribution with an irreducible representation exists and is a geometric distribution. Three numerical examples are provided in this section. In section 4 , a more general situation is considered by adding a discount factor to the equilibrium distributions. And some brief conclusions are stated at the end of the paper.

\section{Equilibrium distributions of the DPH family}

In this section we shall study the equilibrium distributions of $N$ and their properties given that $N$ is of phase-type. One typical example is a stationary discretetime phase-type renewal process. Its occurrence time of the first renewal, the backward and forward recurrence times all follow this type of distributions. See for example Section 3.4 in Latouche [9]. Recently, there has been much attention given to equilibrium distributions and the higher order ones in risk theory literature, see Hesselager et al.[8] and Lin and Willmot [11, 12]. The equilibrium 
distributions play an important role when we study the moments of the time of ruin, the surplus before ruin and the deficit at ruin in classical risk theory. Most recently, Li [10] studied the high order equilibrium distributions of counting random variables and derived a number of interesting general results. Considering the relatively little attention given to the equilibrium distributions in discrete-time risk models, and the practical importance of DPH distributions, we shall focus on investigating the properties of the equilibrium distributions of the DPH family and derive some nice and explicit results within the rest of this paper.

Definition 1 The equilibrium distribution of $N$ is defined as

$$
p_{1}(n):=\frac{\bar{P}(n)}{\mu}=\frac{\sum_{i=n+1}^{\infty} p(i)}{\mu}, \quad n \in \mathbb{N} .
$$

And the lth order equilibrium distribution $(l=2,3, \ldots)$ is then

$$
p_{l}(n):=\frac{\bar{P}_{l-1}(n)}{\mu_{l-1}}=\frac{\sum_{i=n+1}^{\infty} p_{l-1}(i)}{\mu_{l-1}}, \quad n \in \mathbb{N},
$$

where $\mu_{l-1}=\sum_{n=0}^{\infty} \bar{P}_{l-1}(n)$ is the mean of the $(l-1)$ th order equilibrium distribution $\left\{p_{l-1}(n)\right\}_{n \in \mathbb{N}}$.

As a very special case, it is well known that the equilibrium distribution, with any given order, of the geometric distribution is identical to itself. For the general DPH family, we first show that their equilibrium distributions satisfy the following recursion.

Lemma 1 Consider a DPH distribution $\{p(n)\}_{n \in \mathbb{N}}$ with representation $(\overrightarrow{\boldsymbol{\alpha}}, \mathbf{T})$. The survival function and mean of its lth order equilibrium distribution, namely $\bar{P}_{l}(n)$ and $\mu_{l}$, can be expressed as:

$$
\bar{P}_{l}(n)=\overrightarrow{\boldsymbol{\alpha}} \boldsymbol{\Lambda}_{l}(n) \overrightarrow{\mathbf{1}}^{\top}, \quad \mu_{l}=\overrightarrow{\boldsymbol{\alpha}} \boldsymbol{\Pi}_{l} \overrightarrow{\mathbf{1}}^{\top}, \quad l \in \mathbb{N}^{+}, n \in \mathbb{N},
$$

where $\boldsymbol{\Lambda}_{l}(n)$ and $\boldsymbol{\Pi}_{l}$ satisfy the following recursions:

$$
\boldsymbol{\Lambda}_{l}(n)=\frac{\boldsymbol{\Lambda}_{l-1}(n)}{\mu_{l-1}} \mathbf{T}(\mathbf{I}-\mathbf{T})^{-1}, \quad \boldsymbol{\Pi}_{l}=\frac{\boldsymbol{\Pi}_{l-1}}{\mu_{l-1}} \mathbf{T}(\mathbf{I}-\mathbf{T})^{-1}, \quad l \in \mathbb{N}^{+}, n \in \mathbb{N}
$$

with $\boldsymbol{\Pi}_{0}=(\mathbf{I}-\mathbf{T})^{-1}, \mu_{0}=\mu$ and $\boldsymbol{\Lambda}_{0}(n)=\mathbf{T}^{n}$. 
Proof. Using the basic properties of DPH distributions given in Section 1, we have

$$
p_{1}(n)=\overrightarrow{\boldsymbol{\alpha}} \frac{\mathbf{T}^{n}}{\mu} \overrightarrow{\mathbf{1}}^{\top},
$$

and so

$$
\bar{P}_{1}(n)=\sum_{i=n+1}^{\infty} p_{1}(i)=\overrightarrow{\boldsymbol{\alpha}} \frac{\mathbf{T}^{n+1}(\mathbf{I}-\mathbf{T})^{-1}}{\mu} \overrightarrow{\mathbf{1}}^{\top}=\overrightarrow{\boldsymbol{\alpha}} \boldsymbol{\Lambda}_{1}(n) \overrightarrow{\mathbf{1}}^{\top} .
$$

Also,

$$
\mu_{1}=\sum_{n=0}^{\infty} \bar{P}_{1}(n)=\overrightarrow{\boldsymbol{\alpha}} \frac{\mathbf{T}(\mathbf{I}-\mathbf{T})^{-2}}{\mu} \overrightarrow{\mathbf{1}}^{\top}=\overrightarrow{\boldsymbol{\alpha}} \boldsymbol{\Pi}_{1} \overrightarrow{\mathbf{1}}^{\top} .
$$

By induction, we assume (1) and (2) hold for any $l \in \mathbb{N}^{+}$, then

$$
\begin{aligned}
\bar{P}_{l+1}(n) & =\sum_{i=n+1}^{\infty} p_{l+1}(i)=\sum_{i=n+1}^{\infty} \frac{\bar{P}_{l}(i)}{\mu_{l}}=\overrightarrow{\boldsymbol{\alpha}} \sum_{i=n+1}^{\infty} \frac{\boldsymbol{\Lambda}_{l}(i)}{\mu_{l}} \overrightarrow{\mathbf{1}}^{\top} \\
& =\overrightarrow{\boldsymbol{\alpha}} \sum_{i=n+1}^{\infty} \frac{\boldsymbol{\Lambda}_{0}(i) \mathbf{T}^{l}(\mathbf{I}-\mathbf{T})^{-l}}{\prod_{j=0}^{l-1} \mu_{j} \times \mu_{l}} \overrightarrow{\mathbf{1}}^{\top} \\
& =\overrightarrow{\boldsymbol{\alpha}} \frac{\mathbf{T}^{n} \mathbf{T}^{l+1}(\mathbf{I}-\mathbf{T})^{-(l+1)}}{\prod_{j=0}^{l} \mu_{j}} \overrightarrow{\mathbf{1}}^{\top}=\overrightarrow{\boldsymbol{\alpha}} \boldsymbol{\Lambda}_{l+1}(n) \overrightarrow{\mathbf{1}}^{\top}, \quad n \in \mathbb{N}, \\
\mu_{l+1} & =\sum_{n=0}^{\infty} \bar{P}_{l+1}(n)=\overrightarrow{\boldsymbol{\alpha}} \frac{(\mathbf{I}-\mathbf{T})^{-1} \mathbf{T}^{l+1}(\mathbf{I}-\mathbf{T})^{-(l+1)}}{\prod_{j=0}^{l} \mu_{j}} \overrightarrow{\mathbf{1}}^{\top}=\overrightarrow{\boldsymbol{\alpha}} \boldsymbol{\Pi}_{l+1} \overrightarrow{\mathbf{1}}^{\top},
\end{aligned}
$$

which shows (1) and (2) also hold for $l+1$. This completes the proof.

Before we approach to the main result, we need to define a DPH distribution of the second kind that describes the amount of time the underlying Markov chain spent before absorption.

Definition 2 A discrete distribution with p.f. $q(n)$ given by

$$
\begin{aligned}
& q(n)=\overrightarrow{\boldsymbol{\beta}} \mathbf{T}^{n} \overrightarrow{\mathbf{t}}^{\top}, \quad n \in \mathbb{N}^{+}, \\
& q(0)=\beta_{0}+\overrightarrow{\boldsymbol{\beta}} \overrightarrow{\mathbf{t}}^{\top},
\end{aligned}
$$

is called a DPH2 distribution with representation $(\overrightarrow{\boldsymbol{\beta}}, \mathbf{T})$, where $\overrightarrow{\boldsymbol{\beta}}=\left(\beta_{1}, \ldots, \beta_{m}\right)$, $\beta_{0}=1-\sum_{i=1}^{m} \beta_{i}, \mathbf{T}=\left(t_{i, j}\right)_{m \times m}$ is a substochastic matrix and $\overrightarrow{\mathbf{t}}^{\top}=\left(t_{1,0}, t_{2,0}, \ldots, t_{m, 0}\right)^{\top}$ $=(\mathbf{I}-\mathbf{T}) \overrightarrow{\mathbf{1}}^{\top}$. 
Let $Q(n)=1-\bar{Q}(n)=\sum_{i=0}^{n} q(i)$ be the distribution function of a DPH2 distribution $\{q(n)\}_{n \in \mathbb{N}}, \eta^{(l)}$ be its $l$ th factorial mean, and $\hat{q}(z)$ be its p.g.f.. Then we have the following results:

$$
\begin{aligned}
\bar{Q}(n) & =\overrightarrow{\boldsymbol{\beta}} \mathbf{T}^{n+1} \overrightarrow{\mathbf{1}}^{\top}, \quad n \in \mathbb{N}, \\
\eta^{(l)} & =l ! \overrightarrow{\boldsymbol{\beta}}(\mathbf{I}-\mathbf{T})^{-l} \mathbf{T}^{l} \overrightarrow{\mathbf{1}}^{\top}, \quad l \in \mathbb{N}^{+}, \\
\hat{q}(z) & =\beta_{0}+\overrightarrow{\boldsymbol{\beta}}(\mathbf{I}-z \mathbf{T})^{-1} \overrightarrow{\mathbf{t}}^{\top}, \quad|z|<1 .
\end{aligned}
$$

Based on Lemma 1, we have the following main result.

Theorem 1 The lth order equilibrium distribution, $l \in \mathbb{N}^{+}$, of a DPH distribution with representation $(\overrightarrow{\boldsymbol{\alpha}}, \mathbf{T})$ is a DPH2 distribution with representation $\left(\overrightarrow{\boldsymbol{\beta}}_{l}, \mathbf{T}\right)$, where

$$
\overrightarrow{\boldsymbol{\beta}}_{l}=\frac{\overrightarrow{\boldsymbol{\alpha}} \mathbf{T}^{l-1}(\mathbf{I}-\mathbf{T})^{-l}}{\overrightarrow{\boldsymbol{\alpha}} \mathbf{T}^{l-1}(\mathbf{I}-\mathbf{T})^{-l} \overrightarrow{\mathbf{1}}^{\top}} .
$$

Proof. By Lemma 1, we have

$$
\mu_{l}=\overrightarrow{\boldsymbol{\alpha}} \frac{(\mathbf{I}-\mathbf{T})^{-1} \mathbf{T}^{l}(\mathbf{I}-\mathbf{T})^{-l}}{\prod_{j=0}^{l-1} \mu_{j}} \overrightarrow{\mathbf{1}}^{\top}=\frac{\mu^{(l+1)}}{(l+1) ! \prod_{j=0}^{l-1} \mu_{j}},
$$

or equivalently,

$$
\prod_{j=0}^{l} \mu_{j}=\frac{\mu^{(l+1)}}{(l+1) !} .
$$

For any given $l \in \mathbb{N}^{+}$, using this result and based on the definition of the $l$ th order equilibrium distribution and Lemma 1, we have

$$
\begin{aligned}
p_{l}(n) & =\frac{\bar{P}_{l-1}(n)}{\mu_{l-1}}=\frac{\overrightarrow{\boldsymbol{\alpha}} \boldsymbol{\Lambda}_{0}(n) \mathbf{T}^{l-1}(\mathbf{I}-\mathbf{T})^{-(l-1)} \overrightarrow{\mathbf{1}}^{\top}}{\prod_{j=0}^{l-1} \mu_{j}} \\
& =\frac{l ! \overrightarrow{\boldsymbol{\alpha}} \mathbf{T}^{n+l-1}(\mathbf{I}-\mathbf{T})^{-(l-1)} \overrightarrow{\mathbf{1}}^{\top}}{\mu^{(l)}} \\
& =\frac{\overrightarrow{\boldsymbol{\alpha}} \mathbf{T}^{n+l-1}(\mathbf{I}-\mathbf{T})^{-(l-1)} \overrightarrow{\mathbf{1}}^{\top}}{\overrightarrow{\boldsymbol{\alpha}} \mathbf{T}^{l-1}(\mathbf{I}-\mathbf{T})^{-l} \overrightarrow{\mathbf{1}}^{\top}}=\overrightarrow{\boldsymbol{\beta}}_{l} \mathbf{T}^{n} \overrightarrow{\mathbf{t}}^{\top}, \quad n \in \mathbb{N},
\end{aligned}
$$

where

$$
\overrightarrow{\boldsymbol{\beta}}_{l}=\frac{\overrightarrow{\boldsymbol{\alpha}} \mathbf{T}^{l-1}(\mathbf{I}-\mathbf{T})^{-l}}{\overrightarrow{\boldsymbol{\alpha}} \mathbf{T}^{l-1}(\mathbf{I}-\mathbf{T})^{-l} \overrightarrow{\mathbf{1}}^{\top}}
$$


It is obvious that the elements in the vector $\overrightarrow{\boldsymbol{\beta}}_{l}$ sum up to 1 and, by Definition 2 , the distribution $\left\{p_{l}(n)\right\}_{n \in \mathbb{N}}$ is a DPH2 distribution with representation $\left(\overrightarrow{\boldsymbol{\beta}}_{l}, \mathbf{T}\right)$. This completes the proof.

\section{Remarks.}

- Note that the newly defined DPH2 distributions are a type of zero-modified DPH distributions. It can be explained in this way. The time the Markov chain spent before absorption generally is equal to the time to absorption minus 1. Only when the Markov chain initiates from the absorption state or it arrives at the absorption state by one step, the DPH2 random variable takes value 0 . Another method of zero-modifying a DPH random variable is to specify a certain probability for count 0 and then adjusting the existing probabilities of positive counts proportionally. But it is not the case considered here.

- It is well-known that the equilibrium distribution of a continuous phase-type distribution is again of phase-type, and a simple phase-type representation has been found. Thus, the above proved closure property for the discrete case is not surprising.

- It is worth noting that Willmot et al. [18] also considered the equilibrium distributions of DPH family and obtained a similar result with different representation. They concluded that the $l$ th order equilibrium distribution of a DPH $(\overrightarrow{\boldsymbol{\alpha}}, \mathbf{T})$ distribution is a DPH $\left(\vec{\gamma}_{l}, \mathbf{T}\right)$ distribution, where

$$
\vec{\gamma}_{l}=\frac{\overrightarrow{\boldsymbol{\alpha}} \mathbf{T}^{l}(\mathbf{I}-\mathbf{T})^{-l}}{\overrightarrow{\boldsymbol{\alpha}} \mathbf{T}^{l-1}(\mathbf{I}-\mathbf{T})^{-l} \overrightarrow{\mathbf{1}}^{\top}}
$$

and $\vec{\gamma}_{l} \overrightarrow{\mathbf{1}}^{\top} \leq 1$. The representation obtained in Theorem 1 has no difference numerically with the above one, but has a different probabilistic interpretation given below.

We consider a delayed discrete-time $\mathrm{PH}$ renewal process where inter-renewal times i.i.d. follow a DPH $(\overrightarrow{\boldsymbol{\alpha}}, \mathbf{T})$ distribution. Latouche and Ramaswami (1999) Section 3.4 mentioned that the underlying phase process of the discretetime PH renewal process has a generator $\mathbf{T}^{*}=\mathbf{T}+\overrightarrow{\mathbf{t}}^{\top} \overrightarrow{\boldsymbol{\alpha}}$, which includes the two possible ways of transition from $i$ to $j$ with $i \neq j$ : either directly moving from $i$ to $j$ with probability $t_{i j}$ or being absorbed in the state 0 with probability $t_{i}$ first and then restarting instantaneously at state $j$ with probability $\alpha_{j}$. And $\overrightarrow{\boldsymbol{\beta}}_{1}$ given in Theorem 1 is just the stationary vector of $\mathbf{T}^{*}$. When the 
initial distribution of the discrete-time $\mathrm{PH}$ renewal process follows a zerotruncated first-order equilibrium distribution of the inter-renewal times, i.e. $\operatorname{DPH}\left(\overrightarrow{\boldsymbol{\beta}}_{1}, \mathbf{T}\right)$, the PH renewal process becomes a stationary one. It can also be verified that the backward recurrence time of the $\mathrm{PH}$ renewal process at time $n$ follows the first-order equilibrium distribution, and its forward recurrence at time $n$ follows the zero-truncated version.

Based on the properties of the DPH2 distributions listed above and Theorem 1 , we obtain some further results as follows.

Corollary 1 The lth order equilibrium distribution, $\left\{p_{l}(n)\right\}_{n \in \mathbb{N}}$, of a DPH distribution, $\{p(n)\}_{n \in \mathbb{N}}$, with representation $(\overrightarrow{\boldsymbol{\alpha}}, \mathbf{T})$ has the following properties. For $l \in \mathbb{N}^{+}$,

$$
\begin{aligned}
\bar{P}_{l}(n) & =\frac{\overrightarrow{\boldsymbol{\alpha}} \mathbf{T}^{n+l}(\mathbf{I}-\mathbf{T})^{-l} \overrightarrow{\mathbf{1}}^{\top}}{\overrightarrow{\boldsymbol{\alpha}} \mathbf{T}^{l-1}(\mathbf{I}-\mathbf{T})^{-l} \overrightarrow{\mathbf{1}}^{\top}}, \quad n \in \mathbb{N}, \\
\mu_{l}^{(m)} & =\frac{m ! \overrightarrow{\boldsymbol{\alpha}} \mathbf{T}^{m+l-1}(\mathbf{I}-\mathbf{T})^{-(m+l)} \overrightarrow{\mathbf{1}}^{\top}}{\overrightarrow{\boldsymbol{\alpha}} \mathbf{T}^{l-1}(\mathbf{I}-\mathbf{T})^{-l} \overrightarrow{\mathbf{1}}^{\top}}=\frac{m ! l !}{(m+l) !} \frac{\mu^{(m+l)}}{\mu^{(l)}}, \quad m \in \mathbb{N}^{+}, \\
\hat{p}_{l}(z) & =\frac{\overrightarrow{\boldsymbol{\alpha}} \mathbf{T}^{l-1}(\mathbf{I}-\mathbf{T})^{-l}(\mathbf{I}-z \mathbf{T})^{-1} \overrightarrow{\mathbf{t}}^{\top}}{\overrightarrow{\boldsymbol{\alpha}} \mathbf{T}^{l-1}(\mathbf{I}-\mathbf{T})^{-l} \overrightarrow{\mathbf{1}}^{\top}}, \quad|z|<1,
\end{aligned}
$$

where $\mu_{l}^{(m)}$ denotes the mth factorial moment of $p_{l}$ and $\hat{p}_{l}(z)$ is its p.g.f..

Proof. Based on Theorem 1 and properties of the zero-modified DPH distributions, the proof of above results is trivial. Note that the second result is consistent with Theorem 3 in $\mathrm{Li} \mathrm{[10]} \mathrm{and} \mathrm{the} \mathrm{last} \mathrm{one} \mathrm{is} \mathrm{a} \mathrm{much} \mathrm{simplified} \mathrm{version} \mathrm{of} \mathrm{its} \mathrm{The-}$ orem 2.

\section{Limiting equilibrium distributions}

Having proved the property of closure for high order equilibrium distributions of the DPH family, one would naturally want to know what would happen if the order $l$ goes to infinity, given the initial vectors depend on $l$. In the following we shall prove the geometric nature of the limiting equilibrium distributions of the DPH family and present the result in three cases. A numerical example is given for each case for illustration purpose.

At first, we shall consider a discrete distribution with finite support on $\mathbb{N}$. According to Neuts [16] and Latouche and Ramaswami [9], any such distribution is a DPH distribution. We have the following result. 
Theorem 2 Assume that $N$ follows a distribution with finite support on $\mathbb{N}$, and $p(n) \equiv 0$ for all $n>M$, i.e. $M$ is the maximum value $N$ can take. Then the supporting region for the lth order equilibrium distribution, $\left\{p_{l}(n)\right\}_{0 \leq n \leq M}$, is diminishing when $l$ increases, and $\left\{p_{l}\right\}$ converges to a degenerate distribution on 0 when $l$ reaches $M$.

Proof. The proof of the above result is rather simple. The distribution of $N$ has representation $(\overrightarrow{\boldsymbol{\alpha}}, \mathbf{T})$, where

$$
\overrightarrow{\boldsymbol{\alpha}}=\left(p_{1}, p_{2}, \ldots, p_{M}\right) \text { and } \mathbf{T}=\left(\begin{array}{ccccc}
0 & 0 & \cdots & 0 & 0 \\
1 & 0 & \cdots & 0 & 0 \\
0 & 1 & \cdots & 0 & 0 \\
0 & 0 & \cdots & 0 & 0 \\
& & \vdots & & \\
0 & 0 & \cdots & 1 & 0
\end{array}\right)_{M \times M}
$$

Further, matrix $\mathbf{T}$ has only zero eigenvalues. The Cayley-Hamilton theorem implies that $\mathbf{T}^{n}=\mathbf{0}_{M \times M}$ for all $n \geq M$. From Corollary 1 we know that

$$
\bar{P}_{l}(n)=0, \quad \text { for all } l \geq M \text { and } n \in \mathbb{N} .
$$

This completes the proof.

Example 1 Assume that $N$ follows a distribution with finite support on $\mathbb{N}$, with probabilities specified as follows: $\left\{p_{n}\right\}_{n=0}^{10}=(0.4,0.24,0.144,0.086,0.052,0.031$, $0.019,0.011,0.007,0.005,0.005)$.

From the special nature of the matrix $\mathbf{T}$, one can see that the first order equilibrium distribution $p_{1}$ is a zero-modified DPH distribution with an initial vector having at most 9 positive numbers. And similarly, the $l$ th order equilibrium distribution $p_{l}$, for $l \leq 9$, will have an initial vector with at most $10-l$ positive numbers. As $\mathbf{T}^{n}=\mathbf{0}$ for all $n \geq 10$, where $\mathbf{0}$ is a $10 \times 10$ matrix with all zeros, the $l$ th order equilibrium distribution, for any $l \geq 10$, becomes a degenerate distribution with $p_{l}(0)=1$. Table 1 summarises the distributions $\{p\}$ and $\left\{p_{l}\right\}$ for $l=1,2, \ldots, 9$, which shows a consistent trend of degenerating as mentioned. 


\begin{tabular}{l|c|c}
\hline$l$ & $\left\{p_{l}(n)\right\}_{n=0}^{10}$ & $\mu_{l}$ \\
\hline 0 & $\{0.400,0.240,0.144,0.086,0.052,0.031,0.019,0.011,0.007,0.005,0.005\}$ & 1.491 \\
1 & $\{0.402,0.241,0.145,0.087,0.052,0.032,0.019,0.011,0.007,0.003,0.000\}$ & 1.436 \\
2 & $\{0.416,0.248,0.147,0.086,0.050,0.028,0.015,0.007,0.002,0.000,0.000\}$ & 1.299 \\
3 & $\{0.449,0.259,0.145,0.079,0.040,0.019,0.007,0.002,0.000,0.000,0.000\}$ & 1.096 \\
4 & $\{0.502,0.266,0.134,0.062,0.025,0.008,0.002,0.000,0.000,0.000,0.000\}$ & 0.872 \\
5 & $\{0.570,0.265,0.111,0.040,0.011,0.002,0.000,0.000,0.000,0.000,0.000\}$ & 0.663 \\
6 & $\{0.648,0.249,0.081,0.020,0.003,0.000,0.000,0.000,0.000,0.000,0.000\}$ & 0.481 \\
7 & $\{0.732,0.215,0.047,0.006,0.000,0.000,0.000,0.000,0.000,0.000,0.000\}$ & 0.327 \\
8 & $\{0.819,0.162,0.018,0.000,0.000,0.000,0.000,0.000,0.000,0.000,0.000\}$ & 0.199 \\
9 & $\{0.909,0.091,0.000,0.000,0.000,0.000,0.000,0.000,0.000,0.000,0.000\}$ & 0.091 \\
$10+$ & $\{1.000,0.000,0.000,0.000,0.000,0.000,0.000,0.000,0.000,0.000,0.000\}$ & 0.000 \\
\hline
\end{tabular}

Table 1: Equilibrium distributions in Example 1

Secondly, we shall consider a general DPH distribution $\left\{p_{n}\right\}$ with an irreducible representation $(\overrightarrow{\boldsymbol{\alpha}}, \mathbf{T})$, which is equivalent to the matrix $\mathbf{T}+\overrightarrow{\mathbf{t}}^{\top} \overrightarrow{\boldsymbol{\alpha}}$ being irreducible. As shown in Neuts [16], Theorem 2.2.1, a representation that is reducible leads to an irreducible representation after we delete the superfluous rows and columns of $\mathbf{T}$ and the corresponding elements of $\overrightarrow{\boldsymbol{\alpha}}$ and $\overrightarrow{\mathbf{t}}^{\top}$. In this case, the limiting equilibrium distribution of $\left\{p_{n}\right\}$ exists and satisfies the following result.

Theorem 3 Consider a DPH distribution, $\left\{p_{n}\right\}_{n \in \mathbb{N}}$, with an irreducible representation $(\overrightarrow{\boldsymbol{\alpha}}, \mathbf{T})$. Then the limiting equilibrium distribution of $\left\{p_{n}\right\}$ exists and $\lim _{l \rightarrow \infty} \bar{P}_{l}(n)=\delta(\mathbf{T})^{n+1}, n \in \mathbb{N}$, where $\delta(\mathbf{T})$ is the maximum eigenvalue of $\mathbf{T}$.

Proof. We first consider the case where $\mathbf{T}$ is irreducible. As a result of the Perron-Frobenius theorem, also from Neuts [17], we know that $\mathbf{T}$ has a single maximum eigenvalue that is simple and real, denoted by $\delta(\mathbf{T})$. Since $\mathbf{T}$ is a substochastic matrix, we have $\delta(\mathbf{T})<1$. We denote the eigen decomposition (in the complex plane) of $\mathbf{T}$ by $\mathbf{Q} \boldsymbol{\Delta} \mathbf{Q}^{-1}$, where $\boldsymbol{\Delta}=\operatorname{Diag}\left(\delta_{1}, \delta_{2}, \ldots, \delta_{m-1}, \delta(\mathbf{T})\right)$ containing all eigenvalues of $\mathbf{T}$ and $\mathbf{Q}$ is the matrix of corresponding eigenvectors. The eigenvalues $\delta_{1}, \delta_{2}, \ldots, \delta_{m-1}$ are not necessarily all real but their modulus are all less than $\delta(\mathbf{T})$. Then $(\mathbf{I}-\mathbf{T})^{-1}$ would have an eigen decomposition of $\mathbf{Q} \Theta \mathbf{Q}^{-1}$, where $\boldsymbol{\Theta}=\operatorname{Diag}\left(\left(1-\delta_{1}\right)^{-1},\left(1-\delta_{2}\right)^{-1}, \ldots,\left(1-\delta_{m-1}\right)^{-1},(1-\delta(\mathbf{T}))^{-1}\right)$. 
From Corollary 1 we know that

$$
\begin{aligned}
\bar{P}_{l}(n)= & \frac{\overrightarrow{\boldsymbol{\alpha}} \mathbf{T}^{n+l}(\mathbf{I}-\mathbf{T})^{-l} \overrightarrow{\mathbf{1}}^{\top}}{\overrightarrow{\boldsymbol{\alpha}} \mathbf{T}^{l-1}(\mathbf{I}-\mathbf{T})^{-l} \overrightarrow{\mathbf{1}}^{\top}}=\frac{\overrightarrow{\boldsymbol{\alpha}} \mathbf{Q} \Delta^{n+l} \boldsymbol{\Theta}^{-l} \mathbf{Q}^{-1} \overrightarrow{\mathbf{1}}^{\top}}{\overrightarrow{\boldsymbol{\alpha}} \mathbf{Q} \Delta^{l-1} \mathbf{\Theta}^{-l} \mathbf{Q}^{-1} \overrightarrow{\mathbf{1}}^{\top}} \\
= & \frac{\overrightarrow{\boldsymbol{\alpha}} \mathbf{Q} \operatorname{Diag}\left(\frac{\delta_{1}^{n+l}}{\left(1-\delta_{1}\right)^{l}}, \frac{\delta_{2}^{n+l}}{\left(1-\delta_{2}\right)^{l}}, \ldots, \frac{\delta(\mathbf{T})^{n+l}}{(1-\delta(\mathbf{T}))^{l}}\right) \mathbf{Q}^{-1} \overrightarrow{\mathbf{1}}^{\top}}{\overrightarrow{\boldsymbol{\alpha}} \mathbf{Q} \operatorname{Diag}\left(\frac{\delta_{1}^{l-1}}{\left(1-\delta_{1}\right)^{l}}, \frac{\delta_{2}^{l-1}}{\left(1-\delta_{2}\right)^{l}}, \ldots, \frac{\delta(\mathbf{T})^{l-1}}{(1-\delta(\mathbf{T}))^{l}}\right) \mathbf{Q}^{-1} \overrightarrow{\mathbf{1}}^{\top}} \\
= & \frac{\sum_{i=1}^{m-1} \zeta_{i} \frac{\delta_{i}^{n+l}\left(1-\delta_{i}\right)^{l}}{\vartheta_{i}}+\zeta_{m} \frac{\delta(\mathbf{T})^{n+l}}{(1-\delta(\mathbf{T}))^{l}} \vartheta_{m}}{\sum_{i=1}^{m-1} \zeta_{i} \frac{\delta_{i}^{l-1}}{\left(1-\delta_{i}\right)^{l}} \vartheta_{i}+\zeta_{m} \frac{\delta(\mathbf{T})^{l-1}}{(1-\delta(\mathbf{T}))^{l}} \vartheta_{m}}=\sum_{i=1}^{m-1} \omega_{i} \delta_{i}^{n+1}+\omega_{m} \delta(\mathbf{T})^{n+1}, \quad n \in \mathbb{N},
\end{aligned}
$$

where $\zeta_{i}$ and $\vartheta_{i}, i=1,2, \ldots, m$ denote the elements in the vectors $\overrightarrow{\boldsymbol{\alpha}} \mathbf{Q}$ and $\mathbf{Q}^{-1} \overrightarrow{\mathbf{1}}^{\top}$ respectively,

$$
\omega_{i}=\frac{\zeta_{i} \frac{\delta_{i}^{l-1}}{\left(1-\delta_{i}\right)^{l}} \vartheta_{i}}{\sum_{i=1}^{m-1} \zeta_{i} \frac{\delta_{i}^{l-1}}{\left(1-\delta_{i}\right)^{l}} \vartheta_{i}+\zeta_{m} \frac{\delta(\mathbf{T})^{l-1}}{(1-\delta(\mathbf{T}))^{l}} \vartheta_{m}}, \quad i=1,2, \ldots, m-1,
$$

and

$$
\omega_{m}=\frac{\zeta_{m} \frac{\delta(\mathbf{T})^{l-1}}{(1-\delta(\mathbf{T}))^{l}} \vartheta_{m}}{\sum_{i=1}^{m-1} \zeta_{i} \frac{\delta_{i}^{l-1}}{\left(1-\delta_{i}\right)^{l}} \vartheta_{i}+\zeta_{m} \frac{\delta(\mathbf{T})^{l-1}}{(1-\delta(\mathbf{T}))^{l}} \vartheta_{m}} .
$$

One can see that when $l \rightarrow \infty, \omega_{i} \rightarrow 0$, for $i=1,2, \ldots, m-1$ and $\omega_{m} \rightarrow 1$. Therefore, $\lim _{l \rightarrow \infty} \bar{P}_{l}(n)=\delta(\mathbf{T})^{n+1}, n \in \mathbb{N}$.

We then consider the case where the matrix $\mathbf{T}$ is reducible. From Chapter XIII $\S 3$ in Gantmacher [7] and also Neuts [17] we know that the maximum eigenvalue $\delta(\mathbf{T})$ may be multiple. As an example, we assume that the matrix $\mathbf{T}$ has eigenvalues $0 \leq \delta_{1} \leq \delta_{2} \leq \cdots \leq \delta_{m-2}<\delta(\mathbf{T})=\delta(\mathbf{T})<1$, then in the above proof,

$$
\begin{aligned}
\bar{P}_{l}(n) & =\frac{\sum_{i=1}^{m-2} \zeta_{i} \frac{\delta_{i}^{n+l}}{\left(1-\delta_{i}\right)^{l}} \vartheta_{i}+\sum_{j=m-1}^{m} \zeta_{j} \frac{\delta(\mathbf{T})^{n+l}}{(1-\delta(\mathbf{T}))^{l}} \vartheta_{j}}{\sum_{i=1}^{m-2} \zeta_{i} \frac{\delta_{i}^{l-1}}{\left(1-\delta_{i}\right)^{l}} \vartheta_{i}+\sum_{j=m-1}^{m} \zeta_{j} \frac{\delta(\mathbf{T})^{l-1}}{(1-\delta(\mathbf{T}))^{l}} \vartheta_{j}} \\
& =\sum_{i=1}^{m-2} \omega_{i} \delta_{i}^{n+1}+\left(\omega_{m-1}+\omega_{m}\right) \delta(\mathbf{T})^{n+1}, \quad n \in \mathbb{N},
\end{aligned}
$$

where

$$
\omega_{i}=\frac{\zeta_{i} \frac{\delta_{i}^{l-1}}{\left(1-\delta_{i}\right)^{l}} \vartheta_{i}}{\sum_{i=1}^{m-2} \zeta_{i} \frac{\delta_{i}^{l-1}}{\left(1-\delta_{i}\right)^{l}} \vartheta_{i}+\sum_{j=m-1}^{m} \zeta_{j} \frac{\delta(\mathbf{T})^{l-1}}{(1-\delta(\mathbf{T}))^{l}} \vartheta_{j}}, \quad i=1,2, \ldots, m-2,
$$


and

$$
\omega_{j}=\frac{\zeta_{j} \frac{\delta(\mathbf{T})^{l-1}}{(1-\delta(\mathbf{T}))^{l}} \vartheta_{j}}{\sum_{i=1}^{m-2} \zeta_{i} \frac{\delta_{i}^{l-1}}{\left(1-\delta_{i}\right)^{l}} \vartheta_{i}+\sum_{j=m-1}^{m} \zeta_{j} \frac{\delta(\mathbf{T})^{l-1}}{(1-\delta(\mathbf{T}))^{l}} \vartheta_{j}}, \quad j=m-1, m .
$$

One can see that when $l \rightarrow \infty, \omega_{i} \rightarrow 0$, for $i=1,2, \ldots, m-2$ and $\omega_{m-1}+\omega_{m} \rightarrow 1$. As a result, we still have $\lim _{l \rightarrow \infty} \bar{P}_{l}(n)=\delta(\mathbf{T})^{n+1}, n \in \mathbb{N}$. This completes the proof.

Example 2 Let $N$ follow a DPH distribution with representation

$$
\overrightarrow{\boldsymbol{\alpha}}=(0.1,0.2,0.5,0.05,0.15) \text { and } \mathbf{T}=\left(\begin{array}{ccccc}
0.2 & 0.4 & 0 & 0.4 & 0 \\
0 & 0.3 & 0.7 & 0 & 0 \\
0 & 0 & 0.4 & 0 & 0 \\
0 & 0.2 & 0 & 0.8 & 0 \\
0 & 0.2 & 0.1 & 0 & 0.5
\end{array}\right) \text {. }
$$

Note that the matrix $\mathbf{T}$ has five eigenvalues: $0.2,0.3,0.4,0.5$ and 0.8 . Table 2 summarises survival probabilities, $\bar{P}_{l}(n)$, of the $l$ th order equilibrium distribution of $p(n)$ for $n=0,1, \ldots, 10,15,20$, and specially chosen $l$ values as given in the table. At the bottom of the table, corresponding mean values are also provided. It can be seen that $\mu_{l}$ increases as $l$ increases, except the first case of $l=0$. Further, the columns in the table clearly show that when $l$ increases, the equilibrium distribution $\bar{P}_{l}(n)$ converges to $0.8^{n+1}$. When $l=20$, it has converged to at least 8 decimal places. 


\begin{tabular}{c|cccccccc}
\hline$n$ & $l=0$ & 1 & 2 & 3 & 4 & 5 & 10 & 20 \\
\hline 0 & 1.000 & 0.675 & 0.716 & 0.765 & 0.790 & 0.797 & 0.800 & 0.8 \\
1 & 0.670 & 0.457 & 0.524 & 0.594 & 0.626 & 0.636 & 0.640 & 0.64 \\
2 & 0.442 & 0.314 & 0.392 & 0.465 & 0.498 & 0.508 & 0.512 & 0.512 \\
3 & 0.286 & 0.221 & 0.299 & 0.367 & 0.397 & 0.406 & 0.410 & 0.4096 \\
4 & 0.187 & 0.160 & 0.232 & 0.291 & 0.317 & 0.325 & 0.328 & 0.32768 \\
5 & 0.125 & 0.119 & 0.182 & 0.232 & 0.253 & 0.260 & 0.262 & 0.262144 \\
6 & 0.087 & 0.091 & 0.144 & 0.185 & 0.202 & 0.208 & 0.209 & 0.2097152 \\
7 & 0.063 & 0.071 & 0.114 & 0.148 & 0.162 & 0.166 & 0.168 & 0.16777216 \\
8 & 0.047 & 0.055 & 0.091 & 0.118 & 0.129 & 0.133 & 0.134 & 0.13421773 \\
9 & 0.036 & 0.044 & 0.072 & 0.094 & 0.104 & 0.106 & 0.107 & 0.10737418 \\
10 & 0.028 & 0.035 & 0.058 & 0.075 & 0.083 & 0.085 & 0.086 & 0.08589935 \\
15 & 0.009 & 0.011 & 0.019 & 0.025 & 0.027 & 0.028 & 0.028 & 0.02814750 \\
20 & 0.003 & 0.004 & 0.006 & 0.008 & 0.009 & 0.009 & 0.009 & 0.00922337 \\
\hline$\mu_{l}$ & 3.077 & 2.379 & 3.054 & 3.637 & 3.893 & 3.972 & 4.000 & 4.0 \\
\hline
\end{tabular}

Table 2: Equilibrium distributions $\bar{P}_{l}(n)$ in Example 2

At last, we shall consider a special DPH distribution, the convex mixture of $l$ negative binomial distributions, which is of much use in practice. Without loss of generality, we only present the case of $l=2$. As a result of Theorem 3 , we have:

Corollary 2 Assume the distribution of $N$ is a mixture of two negative binomial distributions, $\left\{p^{a}\right\}$ and $\left\{p^{b}\right\}$, in the form of $p(n)=\theta p^{a}(n)+(1-\theta) p^{b}(n), 0<\theta<1$, where the parameters of $\left\{p^{a}\right\}$ and $\left\{p^{b}\right\}$ are $\left(\gamma_{1}, q_{1}\right)$ and $\left(\gamma_{2}, q_{2}\right)$. If $q_{1}<q_{2}$, then the limiting equilibrium distribution of $N$ exists and

$$
\lim _{l \rightarrow \infty} \bar{P}_{l}(n)=q_{2}^{n+1}, \quad n \in \mathbb{N} .
$$

As the proof of the above result is trivial, we rather provide an illustrative numerical example as follows.

Example 3 This example considers a mixture of two negative binomial distributions with parameters $\left(4, q_{1}\right)$ and $\left(3, q_{2}\right)$, i.e. $p(n)=\theta\left(\begin{array}{c}n+3 \\ n\end{array}\right)\left(1-q_{1}\right)^{4} q_{1}^{n}+(1-$ $\theta)\left(\begin{array}{c}n+2 \\ n\end{array}\right)\left(1-q_{2}\right)^{3} q_{2}^{n}, 0<\theta<1$. It is a DPH distribution with seven phases and a representation:

$$
\overrightarrow{\boldsymbol{\alpha}}=\left(\begin{array}{c}
\theta q_{1}^{4}, 4 \theta\left(1-q_{1}\right) q_{1}^{3}, 6 \theta\left(1-q_{1}\right)^{2} q_{1}^{2}, 4 \theta\left(1-q_{1}\right)^{3} q_{1} \\
(1-\theta) q_{2}^{3}, 3(1-\theta)\left(1-q_{2}\right) q_{2}^{2}, 3(1-\theta)\left(1-q_{2}\right)^{2} q_{2}
\end{array}\right)
$$


and

$$
\mathbf{T}=\left(\begin{array}{ccccccc}
q_{1} & 1-q_{1} & 0 & 0 & 0 & 0 & 0 \\
0 & q_{1} & 1-q_{1} & 0 & 0 & 0 & 0 \\
0 & 0 & q_{1} & 1-q_{1} & 0 & 0 & 0 \\
0 & 0 & 0 & q_{1} & 0 & 0 & 0 \\
0 & 0 & 0 & 0 & q_{2} & 1-q_{2} & 0 \\
0 & 0 & 0 & 0 & 0 & q_{2} & 1-q_{2} \\
0 & 0 & 0 & 0 & 0 & 0 & q_{2}
\end{array}\right)
$$

Let $q_{1}=0.3, q_{2}=0.5$ and $\theta=0.3$. According to Corollary 2, we should have $\lim _{l \rightarrow \infty} \bar{P}_{l}(n)=0.5^{n+1}, n \in \mathbb{N}$.

Table 3 summarises survival probabilities, $\bar{P}_{l}(n)$, of the $l$ th order equilibrium distribution of $p(n)$ for $n=0,1, \ldots, 14$, and specially chosen $l$ values as given in the table. At the bottom of the table, corresponding mean values are again provided. It can be seen that $\mu_{l}$ decreases as $l$ increases. And it is obvious that $\bar{P}_{l}(n)$ converges to $0.5^{n+1}$ when $l \rightarrow \infty$. In Table 4 , limiting results $(l=500)$ for different mixture coefficients are put together, which illustrates the result stated in Corollary 2 from another aspect, which is the limiting equilibrium distribution is independent of the mixing coefficient.

\begin{tabular}{l|cccccccccccc}
\hline$n$ & $l=0$ & 1 & 2 & 3 & 4 & 5 & 10 & 20 & 30 & 40 & 50 & 500 \\
\hline 0 & 0.840 & 0.679 & 0.627 & 0.602 & 0.586 & 0.574 & 0.543 & 0.523 & 0.516 & 0.512 & 0.510 & 0.501 \\
1 & 0.623 & 0.440 & 0.384 & 0.357 & 0.340 & 0.327 & 0.294 & 0.274 & 0.266 & 0.262 & 0.260 & 0.251 \\
2 & 0.427 & 0.277 & 0.232 & 0.210 & 0.196 & 0.185 & 0.159 & 0.143 & 0.137 & 0.134 & 0.132 & 0.126 \\
3 & 0.278 & 0.171 & 0.138 & 0.122 & 0.112 & 0.104 & 0.086 & 0.075 & 0.071 & 0.069 & 0.067 & 0.063 \\
4 & 0.176 & 0.103 & 0.081 & 0.070 & 0.063 & 0.058 & 0.046 & 0.039 & 0.036 & 0.035 & 0.034 & 0.032 \\
5 & 0.109 & 0.062 & 0.047 & 0.040 & 0.036 & 0.032 & 0.025 & 0.020 & 0.019 & 0.018 & 0.017 & 0.016 \\
6 & 0.066 & 0.036 & 0.027 & 0.023 & 0.020 & 0.018 & 0.013 & 0.011 & 0.010 & 0.009 & 0.009 & 0.008 \\
7 & 0.040 & 0.021 & 0.016 & 0.013 & 0.011 & 0.010 & 0.007 & 0.005 & 0.005 & 0.005 & 0.005 & 0.004 \\
8 & 0.023 & 0.012 & 0.009 & 0.007 & 0.006 & 0.005 & 0.004 & 0.003 & 0.003 & 0.002 & 0.002 & 0.002 \\
9 & 0.014 & 0.007 & 0.005 & 0.004 & 0.003 & 0.003 & 0.002 & 0.001 & 0.001 & 0.001 & 0.001 & 0.001 \\
10 & 0.008 & 0.004 & 0.003 & 0.002 & 0.002 & 0.002 & 0.001 & 0.001 & 0.001 & 0.001 & 0.001 & 0.000 \\
11 & 0.005 & 0.002 & 0.002 & 0.001 & 0.001 & 0.001 & 0.001 & 0.000 & 0.000 & 0.000 & 0.000 & 0.000 \\
12 & 0.003 & 0.001 & 0.001 & 0.001 & 0.001 & 0.000 & 0.000 & 0.000 & 0.000 & 0.000 & 0.000 & 0.000 \\
13 & 0.001 & 0.001 & 0.000 & 0.000 & 0.000 & 0.000 & 0.000 & 0.000 & 0.000 & 0.000 & 0.000 & 0.000 \\
14 & 0.001 & 0.000 & 0.000 & 0.000 & 0.000 & 0.000 & 0.000 & 0.000 & 0.000 & 0.000 & 0.000 & 0.000 \\
\hline$\mu_{l}$ & 2.614 & 1.817 & 1.573 & 1.453 & 1.377 & 1.322 & 1.182 & 1.095 & 1.064 & 1.049 & 1.039 & 1.004 \\
\hline
\end{tabular}

Table 3: Equilibrium distributions $\bar{P}_{l}(n)$ in Example 3 


\begin{tabular}{l|ccccc|ccccc}
\hline & \multicolumn{6}{|c|}{$\bar{P}(n)$} & \multicolumn{5}{c}{$\bar{P}_{500}(n)$} \\
\hline$n$ & $\theta=0$ & 0.3 & 0.5 & 0.7 & 0.9 & $\theta=0$ & 0.3 & 0.5 & 0.7 & 0.9 \\
\hline 0 & 0.875 & 0.840 & 0.817 & 0.794 & 0.771 & 0.501 & 0.501 & 0.501 & 0.501 & 0.501 \\
1 & 0.688 & 0.623 & 0.580 & 0.536 & 0.493 & 0.251 & 0.251 & 0.251 & 0.251 & 0.251 \\
2 & 0.500 & 0.427 & 0.378 & 0.329 & 0.280 & 0.126 & 0.126 & 0.126 & 0.126 & 0.126 \\
3 & 0.344 & 0.278 & 0.235 & 0.191 & 0.148 & 0.063 & 0.063 & 0.063 & 0.063 & 0.063 \\
4 & 0.227 & 0.176 & 0.142 & 0.109 & 0.075 & 0.032 & 0.032 & 0.032 & 0.032 & 0.032 \\
5 & 0.145 & 0.109 & 0.085 & 0.061 & 0.037 & 0.016 & 0.016 & 0.016 & 0.016 & 0.016 \\
6 & 0.090 & 0.066 & 0.050 & 0.034 & 0.019 & 0.008 & 0.008 & 0.008 & 0.008 & 0.008 \\
7 & 0.055 & 0.040 & 0.029 & 0.019 & 0.009 & 0.004 & 0.004 & 0.004 & 0.004 & 0.004 \\
8 & 0.033 & 0.023 & 0.017 & 0.011 & 0.005 & 0.002 & 0.002 & 0.002 & 0.002 & 0.002 \\
9 & 0.020 & 0.014 & 0.010 & 0.006 & 0.003 & 0.001 & 0.001 & 0.001 & 0.001 & 0.001 \\
10 & 0.011 & 0.008 & 0.006 & 0.004 & 0.001 & 0.000 & 0.000 & 0.000 & 0.000 & 0.000 \\
11 & 0.006 & 0.005 & 0.003 & 0.002 & 0.001 & 0.000 & 0.000 & 0.000 & 0.000 & 0.000 \\
12 & 0.004 & 0.003 & 0.002 & 0.001 & 0.000 & 0.000 & 0.000 & 0.000 & 0.000 & 0.000 \\
13 & 0.002 & 0.001 & 0.001 & 0.001 & 0.000 & 0.000 & 0.000 & 0.000 & 0.000 & 0.000 \\
14 & 0.001 & 0.001 & 0.001 & 0.000 & 0.000 & 0.000 & 0.000 & 0.000 & 0.000 & 0.000 \\
\hline
\end{tabular}

Table 4: Equilibrium distribution $\bar{P}_{500}(n)$ with various $\theta$ values

\section{Equilibrium distributions with discount}

As mentioned in $\mathrm{Li}$ [10], Lin and Willmot [11, 12] and many other papers in risk theory, the first order equilibrium distribution of a claim size distribution can be interpreted as the distribution of the amount of the first drop in the surplus level given there is a drop. It is of important use when studying moments of the time to ruin, the surplus before ruin and the deficit at ruin. In addition, Lin and Willmot [11] also defined another more general form of equilibrium distribution for continuous distributions when some kind of discounting ought to be considered. The new equilibrium distribution can be used in studying the defective renewal equations and the well-known Gerber-Shiu function. In the following, we shall consider its counterpart in the discrete case. Let $0<v \leq 1$ to be the discount factor.

Definition 3 The equilibrium distribution with discount of the distribution $\{p(n)\}_{n \in \mathbb{N}}$ 
is defined as

$$
\bar{G}_{1, v}(n):=\frac{\sum_{i=n+1}^{\infty} v^{i-n} \bar{P}(i)}{\sum_{i=0}^{\infty} v^{i} \bar{P}(i)}, \quad n \in \mathbb{N},
$$

with p.f.

$$
g_{1, v}(n)=\bar{G}_{1, v}(n-1)-\bar{G}_{1, v}(n)=\frac{\sum_{i=n+1}^{\infty} v^{i-n} p(i)}{\sum_{i=0}^{\infty} v^{i} \bar{P}(i)}, \quad n \in \mathbb{N}^{+},
$$

and $g_{1, v}(0)=1-\bar{G}_{1, v}(0)=\frac{\bar{P}(0)}{\sum_{i=0}^{\infty} v^{i} \bar{P}(i)}$.

The lth order equilibrium distribution with discount $(l=2,3, \ldots)$ is

$$
\bar{G}_{l, v}(n):=\frac{\sum_{i=n+1}^{\infty} v^{i-n} \bar{G}_{l-1, v}(i)}{\sum_{i=0}^{\infty} v^{i} \bar{G}_{l-1, v}(i)}, \quad n \in \mathbb{N},
$$

and it has p.f.

$$
g_{l, v}(n)=\bar{G}_{l, v}(n-1)-\bar{G}_{l, v}(n)=\frac{\sum_{i=n+1}^{\infty} v^{i-n} g_{l-1, v}(i)}{\sum_{i=0}^{\infty} v^{i} \bar{G}_{l-1, v}(i)}, \quad n \in \mathbb{N}^{+},
$$

with $g_{l, v}(0)=1-\bar{G}_{l, v}(0)=\frac{\bar{G}_{l-1, v}(0)}{\sum_{i=0}^{\infty} v^{i} \bar{G}_{l-1, v}(i)}$.

From the above definition we can see that the equilibrium distributions discussed in previous sections form a special case of $v=1$, i.e. no discount considered.

When $p(n)$ is $\mathrm{DPH}$, we obtain the following result.

Theorem 4 Let $p(n)$ be a DPH p.f. with representation $(\overrightarrow{\boldsymbol{\alpha}}, \mathbf{T})$. Its lth order equilibrium distribution with discount, $g_{l, v}(n), l \in \mathbb{N}^{+}$, can be expressed in the form of

$$
g_{l, v}(n)=\overrightarrow{\boldsymbol{\beta}}_{l, v} \mathbf{T}^{n} \overrightarrow{\mathbf{t}}^{\top}, \quad n \in \mathbb{N}^{+},
$$

with $g_{l, v}(0)=1-\overrightarrow{\boldsymbol{\beta}}_{l, v} \mathbf{T} \overrightarrow{\mathbf{1}}^{\top}$, where

$$
\overrightarrow{\boldsymbol{\beta}}_{l, v}=\frac{v \overrightarrow{\boldsymbol{\alpha}} \mathbf{T}^{l-1}(\mathbf{I}-v \mathbf{T})^{-l}}{\overrightarrow{\boldsymbol{\alpha}} \mathbf{T}^{l-1}(\mathbf{I}-v \mathbf{T})^{-l} \overrightarrow{\mathbf{1}}^{\top}}
$$

Proof. From Definition 3 we have

$$
\begin{aligned}
\bar{G}_{1, v}(n) & =\frac{\sum_{i=n+1}^{\infty} v^{i-n} \bar{P}(i)}{\sum_{i=0}^{\infty} v^{i} \bar{P}(i)}=\frac{\overrightarrow{\boldsymbol{\alpha}} \sum_{i=n+1}^{\infty} v^{i-n} \mathbf{T}^{i} \overrightarrow{\mathbf{1}}^{\top}}{\overrightarrow{\boldsymbol{\alpha}} \sum_{i=0}^{\infty} v^{i} \mathbf{T}^{i} \overrightarrow{\mathbf{1}}^{\top}} \\
& =\frac{v \overrightarrow{\boldsymbol{\alpha}}(\mathbf{I}-v \mathbf{T})^{-1} \mathbf{T}^{n+1} \overrightarrow{\mathbf{1}}^{\top}}{\overrightarrow{\boldsymbol{\alpha}}(\mathbf{I}-v \mathbf{T})^{-1} \overrightarrow{\mathbf{1}}^{\top}}=\overrightarrow{\boldsymbol{\beta}}_{1, v} \mathbf{T}^{n+1} \overrightarrow{\mathbf{1}}^{\top}, \quad n \in \mathbb{N} .
\end{aligned}
$$


As a result, we obtain

$$
g_{1, v}(n)=\bar{G}_{1, v}(n-1)-\bar{G}_{1, v}(n)=\overrightarrow{\boldsymbol{\beta}}_{1, v} \mathbf{T}^{n} \overrightarrow{\mathbf{t}}^{\top}, \quad n \in \mathbb{N}^{+},
$$

and $g_{1, v}(0)=1-\bar{G}_{1, v}(0)=1-\overrightarrow{\boldsymbol{\beta}}_{1, v} \mathbf{T} \overrightarrow{\mathbf{1}}^{\top}$. By induction, assume that (3) holds for any given $l \in \mathbb{N}^{+}$, then for $l+1$ we have

$$
\begin{aligned}
\bar{G}_{l+1, v}(n) & =\frac{\sum_{i=n+1}^{\infty} v^{i-n} \bar{G}_{l, v}(i)}{\sum_{i=0}^{\infty} v^{i} \bar{G}_{l, v}(i)}=\frac{\overrightarrow{\boldsymbol{\beta}}_{l, v} \sum_{i=n+1}^{\infty} v^{i-n} \mathbf{T}^{i+1} \overrightarrow{\mathbf{1}}^{\top}}{\overrightarrow{\boldsymbol{\beta}}_{l, v} \sum_{i=0}^{\infty} v^{i} \mathbf{T}^{i+1} \overrightarrow{\mathbf{1}}^{\top}} \\
& =\frac{v \overrightarrow{\boldsymbol{\beta}}_{l, v}(\mathbf{I}-v \mathbf{T})^{-1} \mathbf{T}^{n+2} \overrightarrow{\mathbf{1}}^{\top}}{\overrightarrow{\boldsymbol{\beta}}_{l, v}(\mathbf{I}-v \mathbf{T})^{-1} \mathbf{T} \overrightarrow{\mathbf{1}}^{\top}}=\frac{v \overrightarrow{\boldsymbol{\beta}}_{l, v}(\mathbf{I}-v \mathbf{T})^{-1} \mathbf{T}}{\overrightarrow{\boldsymbol{\beta}}_{l, v}(\mathbf{I}-v \mathbf{T})^{-1} \mathbf{T} \overrightarrow{\mathbf{1}}^{\top}} \mathbf{T}^{n+1} \overrightarrow{\mathbf{1}}^{\top} \\
& =\overrightarrow{\boldsymbol{\beta}}_{l+1, v} \mathbf{T}^{n+1} \overrightarrow{\mathbf{1}}^{\top}, \quad n \in \mathbb{N},
\end{aligned}
$$

which gives result (3). It completes the proof.

Moreover, as an extension to Theorem 3 we have the following result for the limiting equilibrium distribution with discount.

Theorem 5 Let $p(n)$ be a DPH p.f. with an irreducible representation $(\overrightarrow{\boldsymbol{\alpha}}, \mathbf{T})$. Its limiting equilibrium distribution with discount exists and $\lim _{l \rightarrow \infty} \bar{G}_{l, v}(n)=v \delta(\mathbf{T})^{n+1}, n \in$ $\mathbb{N}$, where $\delta(\mathbf{T})$ denotes the maximum eigenvalue of $\mathbf{T}$.

Proof. Note that if $\mathbf{T}$ has an eigen decomposition of $\mathbf{Q} \Delta \mathbf{Q}^{-1}$ in the complex plane, then $(\mathbf{I}-v \mathbf{T})^{-1}$ would have an eigen decomposition of $\operatorname{QDiag}((1-$ $\left.\left.v \delta_{1}\right)^{-1},\left(1-v \delta_{2}\right)^{-1}, \ldots,\left(1-v \delta_{m}\right)^{-1}\right) \mathbf{Q}^{-1}$. Having this result, the rest of the proof is very similar to Theorem 3 .

\section{Remarks.}

- Note that Theorem 1 is a special case of Theorem 4 when $v=1$.

- From expression (3) and the fact that $\overrightarrow{\boldsymbol{\beta}}_{l, v} \overrightarrow{\mathbf{1}}^{\top}=v$ we can see that the $l$ th order equilibrium distribution with discount is a mixture of a DPH2 $\left(v^{-1} \overrightarrow{\boldsymbol{\beta}}_{l, v}, \mathbf{T}\right)$ distribution and a degenerate distribution on zero with mixing coefficients $v$ and $1-v$ respectively, which is also shown by Theorem 5 as a limiting case. Comparing with Definition 2, it is actually another approach of zero-modifying DPH distributions. Therefore, corresponding results, including $l$ th factorial mean, p.g.f. etc., can be obtained easily.

In the following, we shall consider another useful variation, which is the $l$ th equilibrium distribution of $g_{1, v}(n)$, denoted by $g_{1, v: l}(n)$. The results for the continuous case can be found in Lin and Willmot [11]. Following Definition 1 and 3 
we can show that

$$
\bar{G}_{1, v: l}(n)=\frac{\sum_{i=n+1}^{\infty} v^{i-n} \bar{P}_{l}(i)}{\sum_{i=0}^{\infty} v^{i} \bar{P}_{l}(i)}, \quad n \in \mathbb{N}
$$

with p.f.

$$
g_{1, v: l}(n)=\frac{\sum_{i=n+1}^{\infty} v^{i-n} p_{l}(i)}{\sum_{i=0}^{\infty} v^{i} \bar{P}_{l}(i)}, \quad n \in \mathbb{N}^{+}
$$

and $g_{1, v: l}(0)=1-\bar{G}_{1, v: l}(0)=\frac{\bar{P}_{l}(0)}{\sum_{i=0}^{\infty} v^{i} \bar{P}_{l}(i)}$.

The following derivation explains the $l=1$ case and higher orders can be proved by induction. By Definition 1 and 3, we have

$$
\begin{aligned}
\bar{G}_{1, v: 1}(n) & =\frac{\sum_{i=n+1}^{\infty} \bar{G}_{1, v}(i)}{\sum_{i=0}^{\infty} \bar{G}_{1, v}(i)}=\frac{\sum_{i=n+1}^{\infty} \sum_{j=i+1}^{\infty} v^{j-i} \bar{P}(j)}{\sum_{i=0}^{\infty} \sum_{j=i+1}^{\infty} v^{j-i} \bar{P}(j)} \\
& =\frac{\sum_{j=n+2}^{\infty} \bar{P}(j) \sum_{i=n+1}^{j-1} v^{j-i}}{\sum_{j=1}^{\infty} \bar{P}(j) \sum_{i=0}^{j-1} v^{j-i}}=\frac{\sum_{j=n+2}^{\infty} \bar{P}(j) \sum_{i=n+1}^{j-1} v^{i-n}}{\sum_{j=1}^{\infty} \bar{P}(j) \sum_{i=0}^{j-1} v^{i-n}} \\
& =\frac{\sum_{i=n+1}^{\infty} v^{i-n} \sum_{j=i+1}^{\infty} \bar{P}(j)}{\sum_{i=0}^{\infty} v^{i-n} \sum_{j=i+1}^{\infty} \bar{P}(j)}=\frac{\sum_{i=n+1}^{\infty} v^{i-n} \bar{P}_{1}(i)}{\sum_{i=0}^{\infty} v^{i-n} \bar{P}_{1}(i)} .
\end{aligned}
$$

As a result, using the previous result for $\bar{P}_{l}(n)$, the $l$ th order equilibrium distribution, $\left\{g_{1, v: l}(n)\right\}$, has the following survival function:

$$
\bar{G}_{1, v: l}(n)=\frac{\sum_{i=n+1}^{\infty} v^{i-n} \overrightarrow{\boldsymbol{\beta}}_{l} \mathbf{T}^{i+1} \overrightarrow{\mathbf{1}}^{\top}}{\sum_{i=0}^{\infty} v^{i} \overrightarrow{\boldsymbol{\beta}}_{l} \mathbf{T}^{i+1} \overrightarrow{\mathbf{1}}^{\top}}=\frac{v \overrightarrow{\boldsymbol{\beta}}_{l}(\mathbf{I}-v \mathbf{T})^{-1} \mathbf{T}}{\overrightarrow{\boldsymbol{\beta}}_{l}(\mathbf{I}-v \mathbf{T})^{-1} \mathbf{T} \overrightarrow{\mathbf{1}}^{\top}} \mathbf{T}^{n+1} \overrightarrow{\mathbf{1}}^{\top},
$$

which is similar to the form of $\bar{G}_{l, v}(n)$. Therefore, we know from previous remarks that $\bar{G}_{1, v: l}(n)$ is again a DPH2 distribution.

\section{Conclusions}

This paper examines one more property of closure for the family of DPH distributions, which is regarding the equilibrium distributions. That is to say, the equilibrium distribution of a DPH distribution with any given order $l$ is still of phase-type, or more precisely, a DPH2 distribution. Several explicit results for the high order equilibrium distributions are presented, which are all of simple form. 
Moreover, the paper also discusses the limiting behavior of the high order equilibrium distributions of the DPH family. Firstly, all discrete distributions with finite support have a degenerate distribution on zero as the limiting equilibrium distribution. Secondly, for a general DPH distribution with an irreducible representation $(\overrightarrow{\boldsymbol{\alpha}}, \mathbf{T})$, its equilibrium distributions converge to a geometric distribution with the maximum eigenvalue of $\mathbf{T}$ as its parameter. As a special case, the mixture of negative binomial distributions has a geometric type limiting equilibrium distribution of which the parameter is inherited from the negative binomial component, within the mixture, with the highest success probability of an experiment. These nice and simple results provide us a big incentive to look for more applications of the higher order equilibrium distributions of the DPH family.

Last but not least, a more general situation is considered by adding a discount factor to the equilibrium distributions. It is shown that the so called equilibrium distributions with discount still have a phase-type structure but with modified probabilities on zero. The limiting equilibrium distribution with discount of an irreducible DPH distribution still exists and has the form of a zero-inflated geometric distribution.

\section{Acknowledgments}

The author is grateful to the referee whose constructive comments have lead to a substantial improvement of the paper. The author would also like to thank Professor David Dickson and Associate Professor Shuanming Li at the University of Melbourne for their helpful suggestions.

\section{References}

[1] Asmussen, S. Phase-type representations in random walk and queuing problems. Ann. Prob. 1992, 20, 772-789.

[2] Bladt, M.; Nielsen, B.F. Moment Distributions of Phase Type. Stoch. Models 2011, 27, 651-663.

[3] Bobbio, A.; Horváth, A.; Scarpa, M.; Telek, M. Acyclic discrete phase type distributions: properties and a parameter estimation algorithm. Perform. Evaluation 2003, 54, 1-32. 
[4] Drekic, S.; Dickson, D.C.M.; Stanford, D.A.; Willmot, G.E. On the distribution of the deficit at ruin when claims are phase-type. Scand. Actuar. J. 2004, 2, 105-120.

[5] Fagiuoli, E.; Pellerey, F. New Partial Orderings and Applications. Nav. Res. Log. 1993, 40, 829-842.

[6] Fagiuoli, E.; Pellerey, F. Preservation of Certain Classes of Life Distributions under Poisson Shock Models. J. Appl. Prob. 1994, 31, 458-465.

[7] Gantmacher, F.R. The theory of matrices, Vol. 2. AMS Chelsea Publishing, 1959.

[8] Hesselager, O.; Wang, S.; Willmot, G.E. Exponential and scale mixture and equilibrium distributions. Scand. Actuar. J. 1994, 20, 125-142.

[9] Latouche, G.; Ramaswami, V. Introduction to matrix analytic methods in stochastic modeling. ASA SIAM, Philadelphia, 1999.

[10] Li, S. The equilibrium distribution of counting random variables. Open J. Discrete Math. 2011, 1, 127-135.

[11] Lin, X.S.; Willmot, G.E. Analysis of a defective renewal equation arising in ruin theory. Insur. Math. Econ. 1999, 25, 63-84.

[12] Lin, X.S.; Willmot, G.E. The moments of the time of ruin, the surplus before ruin, and the deficit at ruin. Insur. Math. Econ. 2000, 27, 19-44.

[13] Maier, R.S.; O'Cinneide, C.A. A closure characterisation of phase-type distributions. J. Appl. Prob. 1992, 29, 92-103.

[14] Nanda, A.K.; Jain, H.; Singh, H. On closure of some partial orderings under mixture. J. Appl. Prob. 1996, 33, 698-706.

[15] Neuts, M.F. Probability distributions of phase type. In: Liber Amicorum Prof. Emeritus H. Florin. University of Louvain 1975, pp. 173-206.

[16] Neuts, M.F. Matrix-geometric solutions in stochastic models: An algorithmic approach. Johns Hopkins University Press, Baltimore, 1981.

[17] Neuts, M.F. The abscissa of convergence of the Laplace-Stieltjes transform of a PH-distribution. Commun. Statist.- Simula. Computa. 1984, 13: 367-373.

[18] Willmot, G.E.; Drekic, S.; Cai, J. Equilibrium compound distributions and stop-loss moments. Scand. Actuar. J. 2005, 1, 6-24. 